\title{
Analysis of the Thoughts of Project-Based Experimental Teaching in Computer Network Course
}

\author{
Lingfang Huang
}

\author{
Jiangxi Science \& Technology Normal University, Nanchang, Jiangxi, 330013 \\ 739960103@qq.com
}

\begin{abstract}
At present, people have entered the information age, and under the background of information age, information technology has become the existence that all walks of life must pay attention to, aiming at the change of market and the demand of society, colleges and universities should also cultivate students' ability to apply information technology, that is, to train students' computer ability. And this is not only to make students get all-round development, but also to improve students' employment competitiveness. In the process of computer network technology teaching, colleges and universities need to pay attention to the importance of practical teaching. In order to integrate the theory teaching and the practice teaching more harmoniously, the university should use the project type The method of experimental teaching improves the effectiveness of talent training in colleges and universities, and this paper also focuses on the analysis of project-based experimental teaching ideas in computer network courses.
\end{abstract}

keywords: computer network

\section{计算机网络课程中的项目式实验教学思路分析}

\author{
黄玲芳
}

\author{
江西科技师范大学 南昌 江西 330010 \\ 739960103@qq.com
}

\section{摘要}

当前人们已经进入了信息化时代，而在信息化时代背景下，信息技术就成为了各行各业都必须引起重 视的存在，针对市场的变化以及社会的需求，高校也应该培养学生的信息技术应用能力，也就是培养 学生的计算机能力。而这不仅仅是为了使学生得到全面发展, 也是为了提高学生的就业竞争力, 在计 算机网络技术教学的过程中, 高校需要注意的是实践教学的重要性。而为了将理论教学和实践教学更 加和谐的融合在一起, 高校应该运用项目式实验教学的方式, 提高高校人才培养的有效性, 而本文也 主要围绕计算机网络课程中的项目式实验教学思路进行分析。

关键词: 计算机网络; 项目式实验教学思路

\section{1. 前言}

随着高科技的快速发展, 计算机网络课程在我国高 等教育当中也越来越重要, 并且计算机网络课程在我国 高校教育中都已经得到了广泛的应用。而计算机网络课 程作为高校计算机专业课程的核心课程, 其重要地位也 具有深远意义。计算机网络课程是指针对学生学习计算 机网络相关的专业方向为基础。学生学习计算机网络课 程也是为了让学生在学习计算机专业课程时打下基础。

\section{2. 计算机网络课程教学中存在的问题}

\section{1 教学内容与实际应用不符}

由于传统的计算机课程知识点比较广泛, 知识内容 比较抽象, 使学生在学习传统的计算机课程当中, 无法 准确的理解该内容的核心。因此，学生对于计算机课程 的学习兴趣也不高。然而大部分学生在学习计算机课程 过程中, 很难将计算机课程的理论知识与计算机实践操 
作联系起来, 大部分学生对于计算机网络课程的理解大 概都还停留在表面的理论基础上, 并不知道如何将计算 机网络课程的理论与实践操作联系。所以, 学生在生活 中遇到有关计算机网络相关问题并不能及时地作出有 效的解决措施。

\section{2 教学方法单一}

计算机网络课程的知识内容主要包含了计算机的 网络理论概念以及对于计算机网络课程的实践操作。所 以, 计算机网络课程相对于其他课程而言会比较特殊。 然而教师再对学生进行计算机网络课程的教学过程当 中, 教师只是一味的将网络计算机网络课程的知识内容 灌输给学生, 使学生只是被动的学习相关知识, 导致学 生在学习计算机网络课程时, 时常感到枯燥乏味, 没有 多大的兴趣, 所以教师对学生进行计算机网络课程的这 种教学方法, 并不利于学生有效的学习计算机网络课 程。

\section{3 缺乏实验教学}

计算机网络课程教学一直以来都是在学习计算机 网络课程理念的基础上, 注重于对计算机网络课程的操 作性。因此, 我国大部分高校对于计算机网络课程教学 当中也会设置实验教学活动。但是由于我国高校教师在 进行计算机网络课程实验教学活动时, 过于注重教学形 式, 按部就班的教导学生操作每个环节, 使学生在进行 计算机网络课程时间操作的时候, 无法依据自己的力量 进行操作。即使在遇到困难时也不会思考, 导致学生在 计算机网络课程实践教学过程当中, 过于依赖老师的教 学。

\section{3. 项目式实验教学在计算机网络课程中的应 用目的}

计算机网络课程中学生所需要学习的不仅是信息 技术的理论知识, 也是计算机技术的应用能力, 虽然计 算机网络课程只是一门基础技能课程, 但是在这门基础 技能课程中学生需要做到基本掌握计算机网络技术, 而 这就要求学生要对计算机网络有一定的了解, 并且能够 将计算机网络知识转化为实践操作, 且真正做到能够解 决计算机网络的实际问题。而之所以提倡高校使用项目 式实验教学法对学生进行计算机网络课程的培养, 也是 因为传统的计算机网络课程教学方法不能做到大幅度 的提升学生的实际操作能力, 甚至对于锻炼学生的实践 操作能力有所阻碍, 且传统的计算机网络课程教学方法 考虑不到学生的学习兴趣的差异以及学生的学习水平 的差异, 对学生采取统一式教学法。但是这样会导致有 一定学习基础的学生时间被浪费, 而学习基础薄弱, 不 够了解计算机网络知识的学生, 则很难跟上教学的进程.
除此之外, 在传统的计算机网络课程教学中, 理论教学 和实践教学是分开来的两个模块, 学生往往是在学习了 理论知识之后再进行实践操作, 因此这样也导致理论与 实践之间有所脱节, 学生很难将理论知识代入到实践操 作中, 以上种种都有可能导致学生厌倦计算机网络课程 学习, 而在失去了学习兴趣之后, 学生的学习效率更加 难以提升。而项目式教学法与传统计算机网络课程教学 方法有着非常大的差异, 项目式教学法能够将理论教学 和实践教学完美的结合在一起, 让学生在了解了理论知 识之后, 迅速地将其运用于实践中, 锻炼自己的实践操 作能力。而且项目式教学法是以学习任务为领导, 学生 完成学习任务的过程, 就是学生锻炼自己的过程。而学 生的学习任务的难度也会不断增加, 当学生能够克服一 个又一个的困难, 渐渐达到一个又一个的学习目标时, 学生的能力就已经有了一定的体现。教师在项目式教学 法中, 所起到的作用只是引导作用, 引导学生去探索和 发现, 并在学生需要的时候给予一定的指导, 但是并不 干涉学生的学习行为以及学生的解决问题的过程, 让学 生能够投入到计算机网络教学中, 并受到计算机网络教 学内容的吸引, 更加深入的探索计算机网络知识, 也不 断提升自己的计算机网络应用能力。

\section{表 1 计算机网络课程项目式实验教学的实践分析}

\begin{tabular}{|c|c|c|}
\hline $\begin{array}{c}\text { 课程的内容与环节 } \\
\text { 设计 }\end{array}$ & 教学内 & $\begin{array}{l}\text { 教学方式与评价 } \\
\text { 方式的设计 }\end{array}$ \\
\hline 通过对学生计算机 & & 较师应 \\
\hline $\begin{array}{l}\text { 基本技能的教学, 使 } \\
\text { 学生能够在未来的 }\end{array}$ & & 训为教 \\
\hline 职业岗位上使用计 & 种办公设备, 在课 & \\
\hline 算机工 & 程内 & 项目实训中强调, \\
\hline $\begin{array}{l}\text { 力, 提高学生计算机 } \\
\text { 操作职业素质。明确 }\end{array}$ & 可增 & 生印象, 同 \\
\hline 教学目标后根据目 & 专业知 & \\
\hline 标明确计算机网络 & 程内容 & , 为今后更 \\
\hline 定课程 & & 变的项目 \\
\hline $\begin{array}{c}\text { 时间关联性, 进而保 } \\
\text { 障教学目标。 }\end{array}$ & & \\
\hline
\end{tabular}

\section{4. 计算机网络课程项目式实验教学的实践分 析}

表 2 计算机网络课程项目式实验教学的实践分析

\begin{tabular}{|c|c|c|}
\hline $\begin{array}{c}\text { 课程的内容与环节设 } \\
\text { 计 }\end{array}$ & $\begin{array}{c}\text { 教学内容的选 } \\
\text { 取 }\end{array}$ & $\begin{array}{c}\text { 教学方式与评价 } \\
\text { 方式的设计 }\end{array}$ \\
\hline 高校教育教学主要是 & 在选择计算机 & 丰富计算机网络 \\
为了学生的学习需求 & 网络课程教学 & 课程教学内容能 \\
以及学生未来的职业 & 内容时, 教师必 & 够提升学生的学 \\
发展而服务, 所以高 & 须要明确计算 & 习热情, 使学生 \\
校的教育教学也必须 & 机网络课程是 & 更加乐于去探索 \\
满足学生的学习需 & 一门实用性课 & 计算机网络课程 \\
求, 并加强与学生未 & 程教学, 因此在 & 教学内容, 而项 \\
来职业发展之间的联 & 使用性课程教 & 目式实践教学法 \\
系性。对不同专业的 & 学中所需要注 & 能够引导学生对 \\
学生, 应该设计不同 & 重的也是知识 & 项目进行探索, \\
的计算机网络课程内 & 的实用性, 而且 & 而理论教学和实 \\
\hline
\end{tabular}




\begin{tabular}{|c|c|c|}
\hline 容, 并在计算机网络 & 因为对于不同 & 践教学也不能分 \\
教学中, 尽可能地体 & 专业的学生而 & 开进行教学, 因 \\
现不同专业的学生的 & 言, 需要掌握的 & 为理论教学和实 \\
专业特性, 并根据不 & 计算机网络知 & 践教学相辅相 \\
同专业的学生设计不 & 识内容不同, 程 & 成, 通过项目式 \\
同程度, 不同类型的 & 度也不同, 所以 & 教学法就能够更 \\
理论内理论教学内容 & 教师也应该根 & 好的整合理论教 \\
和实践教学内容。 & 据不同专业的 & 学和实践教学, \\
& 学生来选择不 & 并且与此同时, \\
& 同难度, 不同侧 & 高校也应该改革 \\
& 重点的知识进 & 计算机网络课程 \\
& 行教学。 & 教学的评价方 \\
& 式, 提高学生平 \\
& 时成绩的占比。 \\
&
\end{tabular}

\section{1 课程的内容与环节设计}

高校教育教学主要是为了学生的学习需求以及学 生未来的职业发展而服务, 所以高校的教育教学也必须 满足学生的学习需求, 并加强与学生未来职业发展之间 的联系性。对不同专业的学生, 应该设计不同的计算机 网络课程内容, 并在计算机网络教学中, 尽可能地体现 不同专业的学生的专业特性, 并根据不同专业的学生设 计不同程度, 不同类型的理论内理论教学内容和实践教 学内容, 不过因为专业多样化, 所以为了节省精力, 教 师可以将同一类别的专业划分为一类, 根据这一类专业 方向设计教学项目, 而在设计计算机网络课程内容之 前, 教师也必须明确教学目标, 教学目标将会成为教学 的引导, 而计算机网络课程教学的目标就是提升学生的 计算机操作能力以及学生的就业优势, 让学生能够成为 一个具备计算机操作能力, 对计算机有一定了解的全面 发展人才。

\section{2 教学内容的选取}

在选择计算机网络课程教学内容时, 教师必须要明 确计算机网络课程是一门实用性课程教学, 因此在使用 性课程教学中所需要注重的也是知识的实用性, 而且因 为对于不同专业的学生而言, 需要掌握的计算机网络知 识内容不同, 程度也不同, 所以教师也应该根据不同专 业的学生来选择不同难度, 不同侧重点的知识进行教 学, 并且尽可能的体现知识的实用性, 让学生能够将学 习到的知识运用到实际中, 并解决现实问题。这样的知 识才能够成为学生的职业能力的一部分, 如果学生仅仅 是掌握了理论知识, 那么学生也无法发挥出理论知识的 价值, 只有在理论知识和实践知识结合的前提下, 学生 才能够运用理论知识进行实践操作。

\section{3 教学方式与评价方式的设计}

丰富计算机网络课程教学内容能够提升学生的学 习热情, 使学生更加乐于去探索计算机网络课程教学内
容，而项目式实践教学法能够引导学生对项目进行探 索, 而理论教学和实践教学也不能分开进行教学, 因为 理论教学和实践教学相辅相成, 通过项目式教学法就能 够更好的整合理论教学和实践教学, 并且与此同时, 高 校也应该改革计算机网络课程教学的评价方式, 提高学 生平时成绩的占比。

\section{5. 项目教学法在具体实践中应该注意的问题}

高校在项目教学法中所需要的是学生每个人都能 够参与到项目教学的实践活动当中, 并且项目教学实践 活动, 它所看重的不是学生在实践活动中学习到的结 果, 而是注重于学生在实践教学过程中学习到了什么, 更注重一种过程。因此, 教师如果要进行项目教学实践 活动，应该注意以下问题：

\section{1 确定项目对于学生的学习过程具有实用 性}

一个项目的确定并不是指由老师的一个单纯的想 法组建的一个活动, 而是老师在通过不断的了解学生的 各种特点, 以及在不断对学生进行教学实践活动中得出 的一个可以全面开展学生, 对于学生有帮助的一种活 动, 并且该项目教学活动是能够使学生在生活中得以运 用。

\section{2 项目活动的形式}

由于高校学生人数比较多, 所以老师在进行项目教 学实践活动当中, 会把学生分成几个小组, 让学生以小 组的形式来进行独立完成活动, 而且这种形式也能够帮 助学生提高团结能力, 锻炼学生的团结意识。

\section{3 教师在项目活动中的指导作用}

当学生在进行项目实践活动中遇到的一些难题, 教 师就要及时的帮助学生解决难题。但是因为项目实践活 动的主体主要是为了帮助学生提高实践技能, 开拓学生 的学习知识, 所以当教师在对学生进行指导时, 不能完 全帮助学生解决难题, 而是要采用一种引导方式, 让学 生独立的去解决遇到的问题。因为只有这样才能够真正 帮助到学生提高实践技能。

\section{6. 结语}

总而言之, 项目式实践教学能够提高人才培养的有 效性, 加强学生的职业能力和职业素养, 也能够使学生 在竞争激烈的市场上保有一份就业优势，所以高校应该 明确项目式实践教学法的教学目标和教学内容, 通过明 
确的教学目标和教学内容来锻炼学生的实践操作能力, 加强学生对计算机网络课程教学内容的理解。

\section{REFERENCES}

[1] Discussion on Experimental Teaching of Computer Network Courses [J]. Cai Xiaoqing. Education and Teaching Forum,2019(39).

[2] Research on the Application of Project-Based Experimental Teaching in Computer Network Courses $[J]$. Cho Jung - Chi. Information Technology and Informatization,2018(04).

[3] Practical application of project-based teaching in computer network teaching [J]. Liao Yu. Curriculum Education Research,2017(26).

[4] An Analysis of Project-Based Experimental Teaching Ideas in Computer Network Courses [J]. Chen Zhen. Computer Knowledge and Technology,2016(25).

[5] Discussion on Project-Based Experimental Teaching in Computer Network Courses [J]. Liu Ruixiao. Computer and Telecom,2016(05).

[6] Analysis of project-based experimental teaching in computer network courses [J]. Zhang Yuwei. Information and Computers (Theoretical Edition),2016(07) 